\title{
EL BALANCE VIDA-TRABAJO EN LAS ORGANIZACIONES DE DIFERENTES PAIISES DEL MUNDO
}

\author{
The work-life balance in the organizations of \\ different countries of the world
}

EPISTEMUS

ISSN: 2007-8196 (electrónico)

ISSN: 2007-4530 (impresa)

\section{Elsy Guadalupe Parada Ruiz ${ }^{1}$ María Del Rocío Soto Flores 2 Daniel González Lomelí 3}

Recibido: 15 de marzo de 2015 Aceptado: 15 de mayo de 2016

Autor de Correspondencia: M.A. Elsy Guadalupe Parada Ruiz Correo: eparada@industrial.uson.mx

\section{Resumen}

Los cambios sociales, demográficos y económicos en el mundo han generado nuevos desafíos para las organizaciones en su búsqueda de la productividad y competitividad. Estos cambios afectan el equilibrio de la vida personal y laboral de los colaboradores incidiendo en su desempeño organizacional. El objetivo del presente estudio fue realizar un análisis de la situación que presentan las organizaciones de diferentes países de Asia, África, Europa y América en relación al balance vida-trabajo y emitir recomendaciones que permitan a las organizaciones implementar políticas realmente efectivas que les generen beneficios en términos de productividad y desempeño.

Los resultados de la revisión permitieron concluir que existen diferencias significativas respecto del entorno cultural, económico y legal de los países analizados. Para que las intervenciones organizacionales que se implementen en la búsqueda del mejoramiento del balance vida-trabajo sean efectivas, es necesario adecuarlas al contexto general del país en el cual se pretenden implementar.

Palabras clave: balance vida-trabajo, intervenciones organizacionales, recursos humanos, contexto global.

\section{Abstract}

Social, demographic and economic changes in the world have created new challenges for organizations in their quest for productivity and competitiveness. These changes affect the balance of work and personal life for employees focusing on organizational performance. The aim of this study was to conduct an analysis of the situation presented by organizations from different countries in Asia, Africa, Europe and America in relation to work-life balance and make recommendations that enable organizations to implement truly effective policies that generate them benefits in terms of productivity and performance.

The results of the review led to the conclusion that there are significant differences in the cultural, economic and legal environment of the countries analyzed. In order to get effective organizational interventions that are implemented in the pursuit of improving work-life balance, it is necessary to adapt the general context of the country in which they intend to implement.

Keywords: work-life balance, organizational interventions, human resources, global context. 


\section{INTRODUCCIÓN}

El balance vida-trabajo (BVT) es un tema que ha venido evolucionando en el transcurso de los años. Los cambios sociales, demográficos y económicos han generado nuevas dinámicas laborales y nuevos retos para los empleados y empleadores. Lejos de pensar que se trata de un fenómeno que debiera resolverse en el ámbito personal, tiene implicaciones significativas en las organizaciones relacionadas con la productividad, competitividad y compromiso de los colaboradores, lo que impacta directamente el ámbito económico y social de las poblaciones.

Las organizaciones en la búsqueda del cumplimiento de sus objetivos están llamadas a colaborar con sus empleados para lograr un equilibrio en sus vidas; esto lleva implícito un beneficio para el empleador que se expresa en un mayor compromiso, lealtad, productividad y satisfacción laboral. El objetivo del presente trabajo es realizar un análisis de la situación que presentan diferentes países de Asia, África, Europa y América en relación al balance vida-trabajo y proponer acciones específicas que generen beneficios para las organizaciones. Se analiza la importancia del balance vida trabajo y su impacto en las personas y las organizaciones. Las diferencias y similitudes encontradas permiten concluir que el contexto cultural, económico y legal determina la efectividad de las intervenciones organizacionales. Un análisis exhaustivo de las condiciones generales del país permitirá adecuar e implementar políticas realmente efectivas en favor de las organizaciones.

\section{GENERALIDADES DEL BALANCE VIDA-TRABAJO}

Para la mayoría de las personas el trabajo constituye uno de los intereses principales en la vida; sin embargo, cuando las demandas laborales obstaculizan otros intereses importantes, se presenta una crisis o conflicto que lleva al estrés y la presión. El trabajo interfiere con la vida personal y viceversa.

La competencia global, el consumismo, los rápidos avances de la tecnología y los cambios en las familias como institución, han incidido en el uso del tiempo, las responsabilidades familiares y las demandas en el trabajo. En la mayoría de las sociedades ha sido la mujer la más afectada por estos cambios en comparación con el hombre [1]. La sobrecarga de trabajo y los conflictos en los roles son predictores del conflicto en la vida de las personas [2]. Greenhaus y Singh [3] definen al BVT como el grado en el cual un individuo está igualmente comprometido y satisfecho con sus roles desempeñados en el trabajo y en su familia (considerando familia y vida personal como sinónimo).

En los últimos años se ha incrementado la atención del BVT en el ámbito académico, político, profesional y en la literatura popular, dada la importancia del tema. Se han estudiado las consecuencias negativas de los conflictos que surgen por la incompatibilidad de las actividades familiares y laborales, entre las que se encuentran mayores riesgos de deterioro de la salud para madres y padres que trabajan, un mal desempeño en la función parental, tensión psicológica, ansiedad, irritación frecuente, depresión, estrés laboral y diversos problemas psicosomáticos [4]. Esta situación no solo afecta a las personas sino también a las empresas; la insatisfacción con el trabajo, el menor desempeño y compromiso con la organización y mayores niveles de ausentismo y rotación, pueden ser efectos derivados de las tensiones provocadas por el desempeño simultáneo de los roles laborales y personales [5].

Los beneficios de implementar políticas amigables son numerosos: personal comprometido y motivado, menores tasas de ausentismo laboral, disminución de la tasa de rotación y por tanto, una mayor retención de empleados competentes, así como una mejora en la productividad [6]. En estudios realizados en América Latina y Europa [6] se ha concluido que no siempre hay coherencia entre las políticas de conciliación vida-trabajo demandadas por los empleados y aquellas puestas en ejecución por las organizaciones.

Los cambios en las estructuras del empleo, como el incremento del empleo a tiempo parcial, han permitido compatibilizar el trabajo y la familia, sin embargo, implica una reducción en lo referente a salarios, seguridad en el empleo y prestaciones no salariales, el desarrollo profesional y las oportunidades de formación. El aumento del empleo informal y las crisis económicas en países de África, Asia y América Latina incrementan la problemática laboral.

La tendencia a las horas de trabajo prolongadas o imprevistas y los horarios poco convencionales como los vespertinos, nocturnos o en fines de semana, en el marco de una economía globalizada en actividad permanente, así como la falta de servicios públicos para trasladarse al lugar de trabajo o la precariedad de los mismo cuando existen, en particular, en los países de bajos ingresos, también aumentan las tensiones entre la vida profesional y la familiar, afectando el bienestar y la productividad de los trabajadores [7].

Desde 2004 la Organización Internacional del Trabajo (OIT) ha realizado estudios relacionados con el tiempo de trabajo. Los países de Oriente Medio, América Latina, el Caribe, Asia y el Pacífico tienen en su mayoría, jornadas semanales superiores a las 48 horas, mientras que Europa y las economías desarrolladas tienen jornadas semanales que van de 35 a 40 horas semanales como máximo, además de un mayor número de días de vacaciones anuales pagadas [8].

La OIT considera necesario establecer un equilibrio aceptable entre las necesidades de las trabajadoras y los trabajadores, los requerimientos de las empresas y las necesidades de la comunidad. El Estado también desempeña una función fundamental en el establecimiento del marco legislativo y de políticas adecuadas, en colaboración con las organizaciones [9]. Según la OIT, existe una justificación económica de las medidas para 


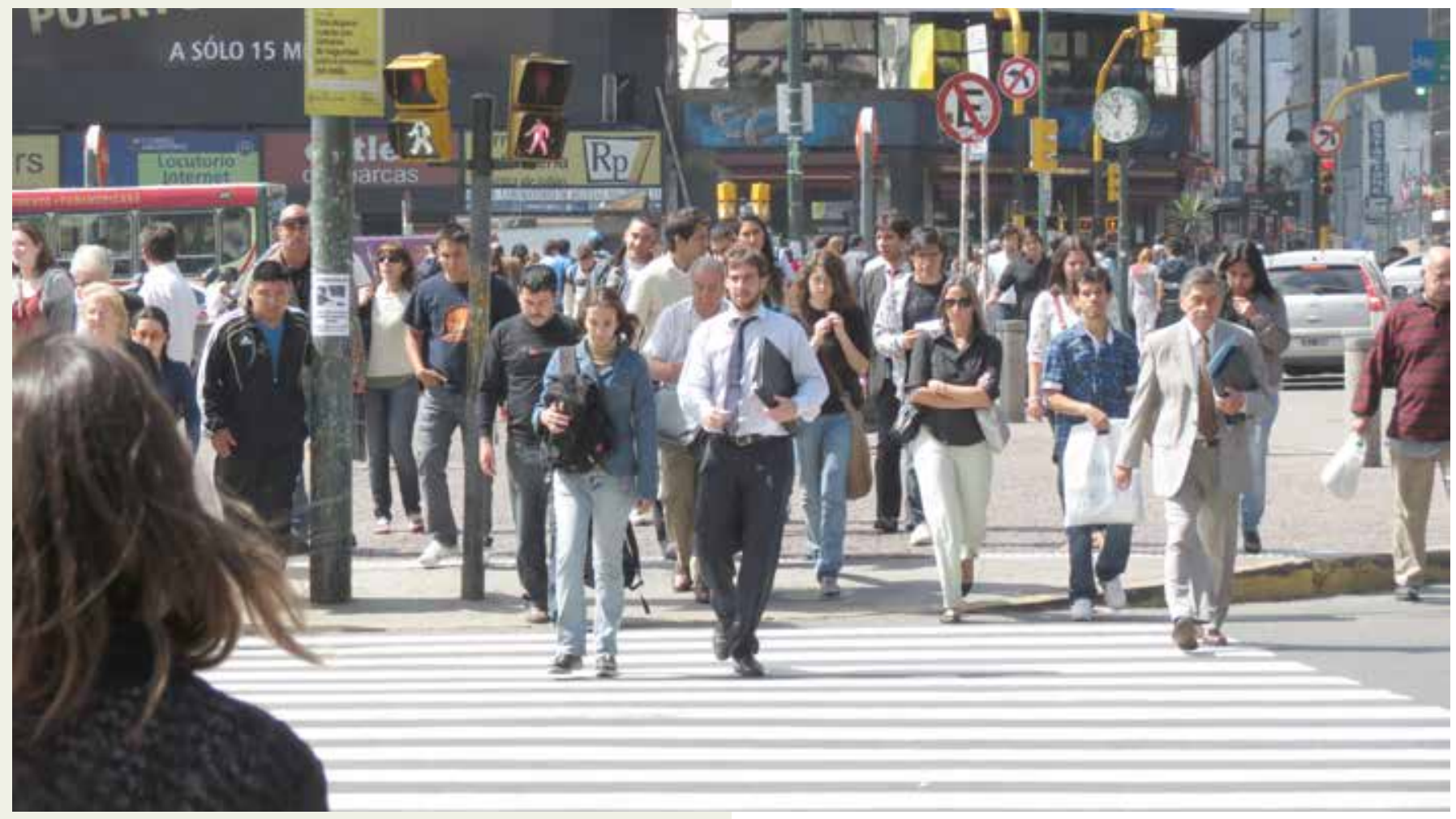

conciliar la vida laboral y familiar relacionada a la eficiencia generada por el uso óptimo del capital humano [7].

\section{EL BALANCE VIDA TRABAJO EN PAÍSES DE ASIA}

El análisis del BVT permite identificar los problemas por imbalance y proponer soluciones, sin embargo el BVT se puede obstaculizar debido a los problemas que se experimentan en países como la India relacionados con las nuevas formas de trabajar derivados de su economía en desarrollo y los aspectos de la cultura tradicional. En los países asiáticos existe conflicto entre la aceptación de la ideología y valores detrás del BVT y la propia cultura [10]. El BVT es visto como sinónimo de prácticas de género y como lucha de las mujeres en un mundo de trabajo dominado por los hombres. Dado que el BVT se percibe en el ámbito de dominio personal, las estrategias de afrontamiento también son individuales y la negociación para la reducción de la jornada es percibida como un signo de debilidad, por lo que los empleados prefieren no hablar del tema.

En Asia, la mayoría de los gerentes hablan acerca de lo bueno que son las políticas familiares, sin embargo, cuando se les pide que modifiquen sus políticas de Recursos Humanos y revelen esta información a sus empleados, muestran intereses diferentes. En la nueva economía de la India, muchos consideran que es una virtud trabajar duro y largas horas hasta el punto de renunciar a su vida personal.

La competencia es feroz y los empleados están dispuestos a trabajar como esclavos. Esto aumenta la incidencia del estrés, los divorcios y los suicidios. Cuando surgen problemas de BVT están dispuestos a sacrificar a la familia por el bienestar laboral y el progreso de sus carreras [1]. Así como en India, el BVT es también poco popular en China, un país sometido a cambios dramáticos en sus estructuras sociales y económicas, donde se otorga especial importancia a los factores que mejoran las habilidades para competir en el mundo de los negocios [11].

\section{EL BALANCE VIDA TRABAJO EN PAÍSES DE EUROPA}

La reconciliación entre el trabajo y la familia es un compromiso que ha llegado a ser crítico en las sociedades europeas para hacer frente a los rápidos cambios tecnológicos y la globalización, una población que envejece, la preocupación por el mercado laboral y el descenso de las tasas de fertilidad [12].

El conflicto vida-trabajo ha impactado potencialmente en el detrimento de la productividad personal, el decremento de la satisfacción de vida y la satisfacción en el trabajo [13], así como padecimientos relacionados con el estrés, incluido los trastornos psicológicos, ansiedad, depresión, agotamiento y abuso del alcohol [14].

Gallie y Russell, citados por McGinnity y Whelan [15], realizaron una investigación en siete países del oeste de Europa y encontraron que el impacto negativo más importante es el incremento en los niveles de presión en el trabajo. Otro estudio como el de Steiber, citado por McGinnity y Whelan [15] resalta el impacto de las demandas laborales derivado de las largas jornadas, las horas impredecibles de trabajo, el trabajo a mediodía, en la noche o el fin de semana. 
De acuerdo a la encuesta de La European Foundation for the Improvement of Living and Working Conditions llevada a cabo en 2007, en la cual participaron 31 países europeos, un promedio de $25 \%$ de los trabajadores en la Unión Europea (UE) percibe que invierten mucho tiempo en el trabajo, el cual llega hasta el $42 \%$ en países que están por integrarse a la Fundación donde las horas trabajadas son mucho mayores.

Por otra parte, un promedio de $34 \%$ de los trabajadores de la UE consideran que invierten muy poco tiempo a su familia. Además, el $36 \%$ de trabajadores en la UE piensan que no tienen suficiente tiempo para actividades sociales y el $51 \%$ estima que no invierte suficiente tiempo en pasatiempos y otras actividades de su interés. En Europa, uno de los principales temas relacionados con el BVT además del estrés, es el relacionado con la sostenibilidad social del trabajo, derivado de la disminución de las tasas de fecundidad de la población [10], así como la problemática por el cuidado de la población adulta mayor.

\section{EL BALANCE VIDA TRABAJO EN PAIISES DE HABLA HISPANA}

En América Latina las políticas dirigidas a las familias carecen de una definición específica, de un campo bien delimitado y de una legislación que la respalde [6]. Más que políticas en beneficio de las familias, existen intervenciones dispersas y no coordinadas [16].

En un estudio realizado a 1,000 compañías españolas y 1,155 latinoamericanas (de Brasil, Chile, Colombia, Perú y El Salvador) de tamaño grande, mediano y pequeño [17] se encontró una diferencia favorable en España relativa a la presencia de políticas que ayudan al BVT, en especial en el caso de los horarios flexibles y el trabajo de tiempo parcial.

Con respecto a la sensibilidad que muestran los directivos hacia las políticas de BVT, los resultados denotan un mayor interés en los países latinoamericanos (73.3\%) que en España (61\%) sin embargo, los directivos de España están haciendo una mejor labor que los países latinoamericanos en el proceso de trasladar sus preocupaciones por los empleados en acciones concretas en la compañía [17]. Los directivos de las pequeñas y medianas empresas hispanas parecen estar más preocupados por las necesidades de sus empleados que las grandes empresas, sin embargo falta incluir estos temas en sus agendas de trabajo.

\section{EL BALANCE VIDA TRABAJO EN PAÍSES DE ÁFRICA}

En un estudio cualitativo realizado en Nigeria [18] se encontró que la percepción de las personas con respecto a las prácticas de BVT, están relacionadas en su mayoría con el factor macro-ambiental del país, como son los aspectos políticos, económicos y retos sociales que se enfrentan. En el estudio los empleados coincidieron en que la corrupción es un gran problema que enfrenta la población.

Los empleados entrevistados expresaron que los fondos asignados en la agenda gubernamental para las iniciativas relacionadas con la promoción del BVT como los apoyos sociales, las facilidades para cuidado de menores, permisos y subsidios eran la mayor parte de las veces malversados en los sectores públicos y privados. De igual forma los empleados expresaron su descontento con la pobre infraestructura social derivada del mal liderazgo de sus gobernantes. La falta de agua potable, casas, suministro de electricidad, buenas carreteras y sistemas de transportes son la generalidad. Las autopistas en pésimas condiciones y la falta de semáforos dificultan los traslados a las organizaciones generando embotellamientos, pérdida de tiempo y estrés en los empleados.

Bajo estas condiciones sociales, no es posible lograr un BVT [18], [19]. Otro factor importante que derivó del estudio es la sobrecarga de los roles, la cual es la principal causa de falta de BVT en las mujeres de Nigeria. Las diferencias de género relativas a los derechos laborales también son significativas [20]. Además, de acuerdo a lo expresado por los empleados entrevistados, el conflicto es parte de sus vidas debido a que los empleadores tienen la mentalidad de que el conflicto vida-trabajo es un problema personal de los empleados, sin embargo se muestran satisfechos con recibir al menos lo indispensable para vivir, por lo que se ha desarrollado una resistencia personal psicológica y física a la problemática.

\section{CONCLUSIONES}

Se encontró que el aspecto cultural es de gran importancia en la implementación de prácticas amigables en las organizaciones; mientras los empleados en Europa y América dicen tener necesidad de mejorar su BVT, en Asia hay resistencia a tomar las prácticas que existen debido al temor de que sea considerado como un aspecto laboral negativo. En África los aspectos culturales están sumamente arraigados y se afronta el conflicto de una manera personal.

Los horarios de trabajo flexible y reducidas como parte de las medidas básicas en la búsqueda del BVT son poco utilizados en países de Asia y África y poco fomentados por las organizaciones, mientras que en Europa se presentan condiciones laborales aceptables y beneficios mayores, con un creciente interés por parte de las organizaciones. En América no existe una formalización de las medidas y éstas se implementan de manera informal y desorganizada, lo que puede afectar el desempeño laboral y el contexto familiar.

Las políticas públicas en países de Europa han reconocido la importancia del BVT tratando de mejorar las condiciones legales, de infraestructura, seguridad laboral e inversión en beneficio de los trabajadores, lo mismo sucede en todos los países desarrollados, situación poco favorecida en los países de África, América y Asia, donde existe poco interés por parte de las autoridades en establecer un marco referencial legal a partir del cual se fomente el BVT.

La implementación de medidas por parte de las organizaciones en la búsqueda del BVT de sus 
colaboradores y su respectivo beneficio para las empresas deberá estar precedido de un análisis exhaustivo de las condiciones económicas de la sociedad en términos de infraestructura y servicios además de una revisión de la situación legal laboral que permita fomentar los beneficios que se tienen establecidos y proponer iniciativas acordes a la realidad actual. La herencia cultural es un predictor del comportamiento de los empleados. Si bien es cierto que los aspectos culturales están sumamente arraigados en las personas, el apoyo y orientación puede llevar a una reflexión que genere un cambio paulatino en las conductas.

Es fundamental la promoción del BVT en las organizaciones independientemente del país, región, contexto general y tipo de organización. Como se visualiza en la figura 1, las políticas y prácticas organizacionales deberán estar siempre encaminadas a fomentar dicho balance. Las organizaciones no son un ente aislado de la sociedad, se encuentran ubicadas en un contexto económico, legal y cultural específico y su reconocimiento es fundamental para la implementación de políticas acorde a las necesidades de los trabajadores. Es por ello que las organizaciones deben implementar políticas y programas encaminados a mejorar el BVT considerando la interrelación de los contextos que le afectan.

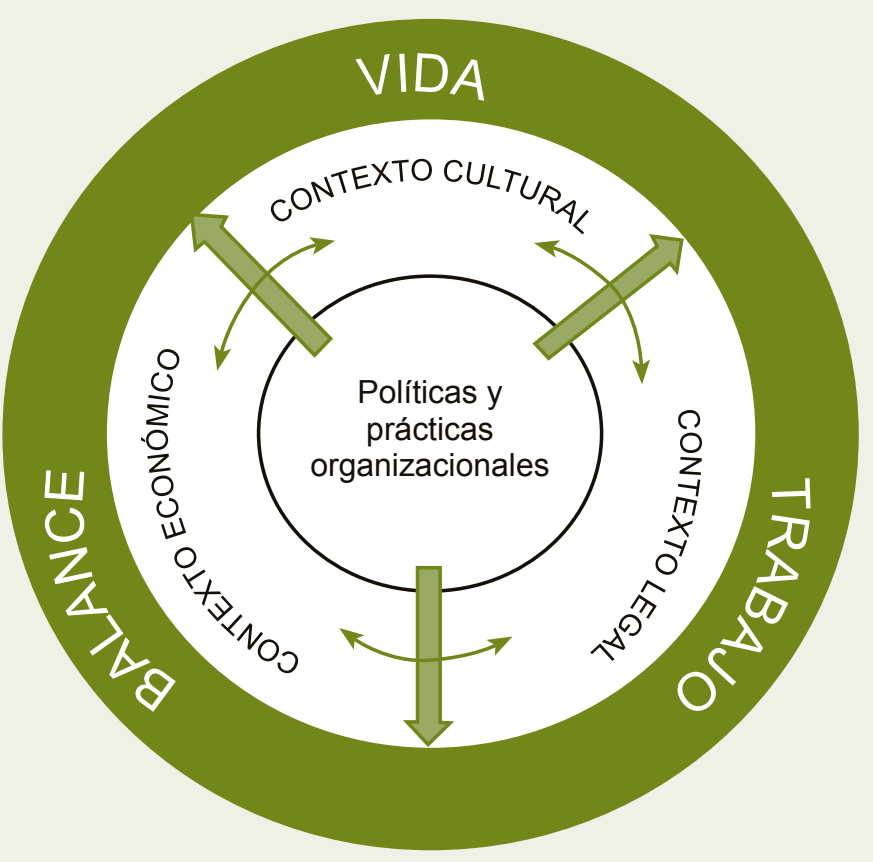

Figura 1. Las políticas y prácticas organizacionales dirigidas al fomento del balance vida-trabajo y sus contextos. of Management, vol.26, no.5, pp.1031-1054, 2000.

[3] J. H. Greenhaus and L. Singh "Work-Family Linkages, A Sloan Work and Family Encyclopedia Entry", Chestnut Hill, MA: Boston College, February 2003.

[4] M. R. Frone, M. Russell and M. Lynne, "Antecedents and outcomes of work-family conflict: testing a model of a workfamily interface", Journal of Applied Psychology, vol.77, no.1, pp.75-78, 1992.

[5] J. H. Greenhaus and N. J. Beutell, "Sources of Conflict Between Work and Family Roles", Academy of Management Review, vol.10, no.1, pp.76-88, 1985.

[6] CEPAL, "Familias y políticas públicas en América Latina: una historia de desencuentros", Santiago de Chile. Publicación de las Naciones U nidas, 2007.

[7] OIT, "Conciliación del trabajo y la vida familiar", 312ª. Reunión, Ginebra, Suiza, 2011.

[8] OIT, “El tiemo de trabajo en el siglo XXI”, Ginebra, Suiza, 2011.

[9] OCDE, "Doing better for families," OECD Publishing. Consultado en: http://dx.doi.org/10.1787/9789264098732en.

[10] S. Lewis, R. Gambles and R. Rapoport, "The constraints of a work-life balance approach:an international perspective", International Journal of Human Resource Management, vol.18, no.3, pp.360-373, March 2007.

[11] K. Chan and T. Wyatt, "Quality of Work Life: A Study of Employees in Shanghai, China", Asia Pacific Business Review, vol.13, no.4, pp.501-517, 2007.

[12] OCDE,"Balancing Work and Family Life: helping parents into paid employment", OECD Employment Outlook, 2001.

[13] J.C. Gornick and M.K. Meyers, "Families that work-Policies for reconciling parenthood and employment", New York: Russell Sage Foundation, 2003.

[14] T. D. Allen, D. E. L. Herst, C.S. Bruck and M. Sutton, "Consequences associated with work-to-family conflict: A review and agenda for future research", Journal of Occupational Health Psychology, vol.5, no. 2, pp.278-308, 2000.

[15] F. McGinnity and C. Whelan, "Comparing Work-Life Conflict in Europe: Evidence from the European Social Survey", Social Indicators Research, vol.93, pp.433-444, 2009.

[16] I. Arriagada, "Familias latinoamericanas. Diagnóstico y políticas públicas en los inicios del nuevo siglo", seire Políticas Sociales, vol.57 (LC/L.1652-P), Santiago de Chile, Comisión Económica para América Latina y el Caribe (CEPAL). Publicación de las Naciones Unidas, 2001.

[17] S. Idrovo, C. León and M. Grau, “Comparing work-life balance in Spanish and Latin-American countries", European Journal of Training and Development, vol.36, no.2, pp.286-307, December 2011.

[18] B. Akanji,"Realities of work life balance in Nigeria: perceptions of role confict and coping beliefs", Business, Management and Education, vol.10, no.2, pp.248-263, December 2012.

[19] T. D. Allen, "Family-Supportive Work Environments: The Role of Organizational Perceptions", Journal of Vocational Behavior, no.58, pp.414-435, 2001.

[20] L. T. Eby, W. J. Casper, A. Lockwood, C. Bordeaux and A. Brinley, "Work and family research in IO/OB: Content analysis and review of the literature (1980-2002)", Journal of Vocational Behavior, vol. 66, pp.124-197, February 2004.

\section{BIBLIOGRAFÍA}

[1] V. Chandra, "Work-life balance: eastern and western perspectives", The International Journal of Human Resource Management, vol.23, no.5, pp.1040-1056, March 2012.

[2] D. S. Carlson and K. M. Kacmar, "Work-Family Conflict in the Organization: Do life role values make a difference?", Journal 